\title{
AN UNCOMMON PRESENTATION OF MILLER FISHER SYNDROME
}

\author{
Nameirakpam Dhanachand Singh1, Lairenjam Sushima Devi², Thounaojam Rameshchandra3 , Laishram Nirupama4, \\ Akoijam Bijayarani ${ }^{5}$
} 1Senior Resident, Department of General Medicine, Jawaharlal Nehru Institute of Medical Sciences, Porompat, Imphal.
2Junior Resident, Department of Paediatrics, DY Patil, Hospital, Kolhapur.
3Senior Resident, Department of General Medicine, Jawaharlal Nehru Institute of Medical Sciences, Porompat, Imphal.
4Junior Resident, Department of General Medicine, Jawaharlal Nehru Institute of Medical Sciences, Porompat, Imphal.
5Junior Resident, Department of General Medicine, Jawaharlal Nehru Institute of Medical Sciences, Porompat, Imphal.

\section{ABSTRACT}

\section{BACKGROUND}

Miller Fisher Syndrome (MFS) is a localised variant of Guillain-Barré Syndrome (GBS), which consists of ophthalmoplegia, ataxia and areflexia without any weakness. ${ }^{1}$ Most of the patients with MFS present with at least two features and have in support an elevated Cerebrospinal Fluid (CSF) protein and characteristic autoantibody. Bickerstaff's Brain Stem Encephalitis (BBE) is a variant of MFS characterised by alteration in consciousness, paradoxical hyperreflexia, ataxia and ophthalmoparesis. ${ }^{2}$ The frequency of BBE variant is $10 \%$ of that of MFS. ${ }^{3}$ Some MFS cases may progress to otherwise classic GBS. Hereby, we report a case who initially presented with ataxia and ophthalmoplegia, then subsequently developed areflexia with no history of fever, upper respiratory tract infection and diarrhoeal illness. Cerebrospinal fluid study and Nerve conduction velocity study supported our clinical diagnosis. Considerable improvement in symptoms including ataxia and ophthalmoplegia were seen after third cycles of plasmapheresis. Though gradual complete recovery is the usual outcome, some life-threatening complications can occur if not treated properly at the earliest time. So proper history, clinical and investigations must be worked up to diagnose early as some MFS can progress to classic GBS and to differentiate from BBE.

\section{KEYWORDS}

Miller Fisher Syndrome, Guillain-Barré Syndrome, Bickerstaff s Brain Stem Encephalitis.

HOW TO CITE THIS ARTICLE: Singh ND, Devi LS, Rameshchandra T, et al. An uncommon presentation of Miller Fisher syndrome. J. Evolution Med. Dent. Sci. 2017;6(21):1740-1741, DOI: 10.14260/Jemds/2017/382

\section{BACKGROUND}

\section{Case Report}

A 53 years old woman with no known comorbidities came with chief complaints of drooping of both upper eyelid with double vision since 5 days, unsteadiness while walking since 4 days, nasal intonation and nasal regurgitation for a duration of 2 days.

On admission, patient was afebrile with normal mentation. Her vitals were normal. Neurological Examination revealed - bilateral ptosis; bilaterally dilated pupils not reacting to light; no nystagmus; consensual and accommodation light reflex absent; normal fundus ; absent external ocular movements in all the directions; power $-5 / 5$ in all limbs; tone - Hypotonia; deep tendon reflexes and abdominal reflexes absent; plantar bilaterally mute; impaired tandem walking test. All other systemic examinations were normal.

\section{Investigation}

Complete haemogram, biochemical parameters were within normal limits. Electrocardiogram and echocardiogram were normal. Chest x-ray PA view was normal. Both NCCT brain and MRI brain were normal. HIV, HbsAg, HCV and VDRL

Financial or Other, Competing Interest: None.

Submission 21-01-2017, Peer Review 26-02-2017,

Acceptance 04-03-2017, Published 13-03-2017.

Corresponding Author:

Nameirakpam Dhanachand Singh,

Shanti Clinic, 139,

Masjid Road, Paona Bazar,

Imphal West-795001,

Manipur.

E-mail: dhanazsingh521@gmail.com

DOI: $10.14260 /$ jemds $/ 2017 / 382$

\section{(c) $($ ) $\$$}

serology were negative. Lumbar puncture of CSF: WBC $4 / \mathrm{mm}^{3}$, Protein - $74 \mathrm{mg} / \mathrm{dL}$, Glucose - $60 \mathrm{mg} / \mathrm{dL}$; Nerve conduction study showed Motor - Normal, Sensory - All nerve has absent SNAP's and F wave latency.

History, clinical examination and investigation findings were suggestive of Miller Fisher Syndrome and treatments started with 5 cycle of Plasmapheresis, which were given over a period of 15 days. Patient considerably improved, ptosis slightly decreased and started walking with no unsteadiness after $3^{\text {rd }}$ cycle of plasmapheresis. Gradual improvement of symptoms was seen day by day and after 3 weeks patient was discharged from the hospital to be followed up in outpatient department.

\section{DISCUSSION}

Miller Fisher Syndrome (MFS) also called Fisher's syndrome are the clinical triad of ophthalmoplegia, ataxia and areflexia. ${ }^{4}$ MFS is named after Dr. C. Miller Fisher who described it in 1956 as a limited variant of ascending paralysis, i.e. GuillainBarré Syndrome (GBS). Though, MFS represents 5 to $10 \%$ of GBS cases in Western countries, it is more common in Eastern Asia, accounting for up to $25 \%$ of Japanese cases. ${ }^{5}$ Miller Fisher syndrome occurs in more men than women by a ratio of approximately 2:1.6 The mean age of onset of MFS is 43.6 years, though onset has been documented in individuals between the ages of 13 and 78 years. ${ }^{7}$

Patients typically seek medical attention because of a rapid decrease in vision over days and/or difficulty walking. These changes are frequently preceded by a viral or diarrheal illness 1 to 4 weeks earlier. Slurred speech, difficulty swallowing and abnormal facial expression with inability to smile or whistle may also occur. Examination shows poor balance and coordination of the hands as well as loss of deep tendon reflexes and eye muscle weakness. 
Nerve conduction test may show diminished activity of nerves that carry sensory information to the spinal cord and brain. The most consistent electrophysiological findings in MFS are reduced sensory nerve action potentials and absent $\mathrm{H}$ reflexes. More variability is seen with $\mathrm{F}$ waves and various investigations involving cranial structures. Magnetic Resonance (MRI) or other imaging of the brain and/or spinal cord are usually normal. Cerebrospinal fluid protein is often elevated (albuminocytologic dissociation). Pure Fisher syndrome is uncommon with many patients going on to develop the prominent widespread weakness of GBS.

Ataxia is primarily noted during the patient's gait, typically in the trunk and with lesser involvement of the limbs. Motor strength is characteristically spared. Anti-GQ1b antibodies, activated by certain strains of Campylobacter jejuni, have a relatively high specificity and sensitivity for MFS. Dense concentrations of GQ1b ganglioside are found in the oculomotor nerve (cranial nerve III), trochlear nerve (cranial nerve IV) and abducens nerve (cranial nerve VI) of patients with MFS, which may explain the relationship between anti-GQ1b antibodies and ophthalmoplegia.6,8 Titers of anti-GQ1b antibodies in CSF that are greater than 1:40 are specific for MFS.

The cause(s) of Fisher's syndrome is not completely understood. The waddling, duck-like gait is likely due to the loss of a fat-rich insulating material called myelin around nerves, designated as $1 \mathrm{~A}$, that innervate the major sensory organ of muscle called the muscle spindle. As the clinical course progresses, other sensory fibers can be involved as well as motor and autonomic fibers that respectively innervate muscles that move the eyes and face and control function of the eye, pupil and the bladder. Multiple lines of evidence support an autoimmune mechanism in which the preceding/triggering infection stimulates production of an antibody that reacts to a sugar found on both the surface of infectious organism and the peripheral nerve causing demyelination and loss of function of the nerve.

Fortunately, this disorder is often short lived, progressing for only a few weeks and then improving. Anti-GQ1b antibody is one of the key factors in the pathogenesis of MFS, especially for ophthalmoplegia and it is a useful marker in diagnosis of MFS, but it was not done in our case because of unavailability of the test. The combination of absent or reduced sensory responses on clinical electrophysiology testing and elevated protein with a normal white blood cell in CSF could lead to the diagnosis of MFS. ${ }^{9}$ MFS symptoms can signal the beginning of GBS with breathing difficulties, which may sometimes lead to respiratory depression, so patients are often hospitalised for observation. In pure MFS, a near full recovery typically occurs within 2 - 3 months. In rare cases when symptoms substantially impair function, various treatments that limit or neutralise immune system activity may be considered. These include high dose intravenous immunoglobulin (IVIG) or plasmapheresis (a procedure in which antibodies are removed from the blood) and supportive care. 10

\section{CONCLUSION}

To conclude, a thorough proper history taking, complete physical examination and proper relevant investigations are required to be done so as to diagnose these rare but interesting neurologic disorder which is known as Miller Fisher Syndrome. Though gradual complete recovery is the usual outcome, life-threatening complications can occur if not treated properly at the earliest time. Intravenous immunoglobulin administration or plasmapheresis with conservative management gradually improves the symptoms.

\section{REFERENCES}

[1] Fisher CM. An unusual variant of acute idiopathic polyneuritis (syndrome of ophthalmoplegia, ataxia and areflexia). N Engl J Med 1956;255(2):57-65.

[2] Bickerstaff ER, Cloake PC. Mesencephalitis and rhombencephalitis. Br Med J 1951;2(4723):77-81.

[3] Ito M, Kuwabara S, Odaka $M$, et al. Bickerstaff's brainstem encephalitis and fisher syndrome form a continuous spectrum: clinical analysis of 581 cases. J Neurol 2008;255(5):674-82.

[4] Jacobs BA, van Doorn PA. ISNO >Neuromuscular info $>$ Disorders and diagnostics $>$ Miller fisher syndrome. 2005.

[5] Overell JR, Hsieh ST, Odaka M, et al. Treatment for fisher syndrome, bickerstaff's brainstem encephalitis and related disorders. Cochrane Database Syst Rev 2007;(1):CD004761.

[6] Berlit P, Rakicky J. The miller fisher syndrome. Review of literature. J Clin Neuroophthalmol 1992;12(1):5763.

[7] Mori M, Kuwabara S, Fukutake T, et al. Clinical features and prognosis of miller fisher syndrome. Neurology 2001;56(8):1104-6.

[8] Winer JB. Guillain barre syndrome. Mol Pathol 2001;54(6):381-5.

[9] Sever M, Aksay E, Gulec F. The diagnosis is made only with suspicion: Miller fisher syndrome. Hong Kong Journal Emergency Medicine 2011;18(6):428-31.

[10] NINDS Miller fisher syndrome information page. https://www.ninds.nih.gov/Disorders/All Disorders/Miller-Fisher-Syndrome-Information-Page. 